\title{
METROLOGY OF ELECTROMAGNETIC INTENSITY MEASUREMENT IN NEAR FIELD
}

DOI: 10.12776/QIP.V17I1.79

\author{
JOZEF SLÍŽIK, RENÉ HARŤANSKÝ
}

\section{INTRODUCTION}

Measurement of the electromagnetic field has its own specifics, which are mainly in the fact that it is necessary to measure two of the vectors: electrical field marked as $\overline{\boldsymbol{E}}$ and magnetic field marked as $\overline{\boldsymbol{H}}$. Measurement of the far field is sufficiently known problem. It is know that problems, which are occurring in the near field area, are associated with the frequency dependent sensors of the field and with their geometric arrangement (Hartanský \& Bittera, 2001; Petrik \& Palfy, 2009). In most cases we can consider far field to be homogeneous on the volume (surface) enclosed with the sensor of the electric field.

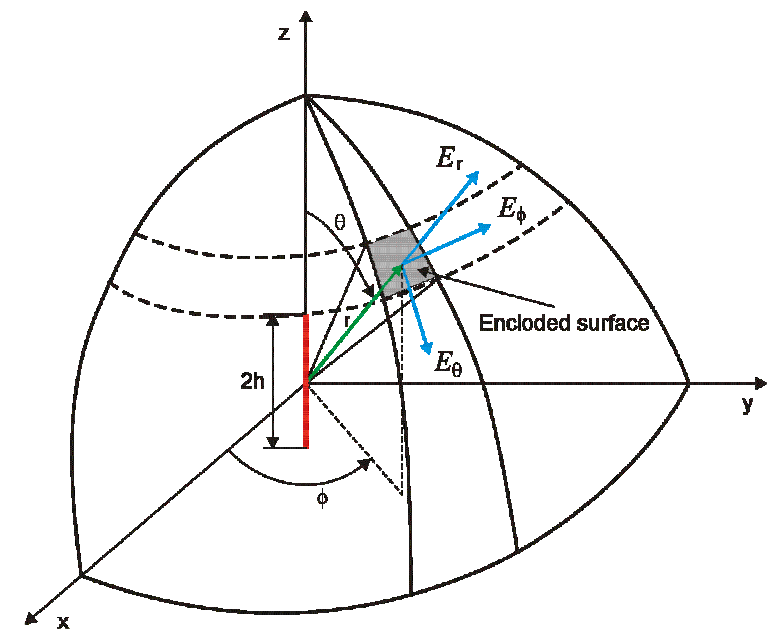

Figure 1 - Components of electromagnetic field of electric source

In this case the sensor does not bring into the result of measurement an error, which is dependent from integral properties of the sensor. This result we can't generalize on the measure in the near field.

In this article we deal with error, which arises from measurement of the source of the electromagnetic field $\bar{E}$. Measurement is realized in the near zone of the dipole source, i.e. if $k \cdot r \ll 1$ ( $k$-wave number, $r$-distance) and must apply 
$r \ll \frac{\lambda}{2 \pi}$ ( $\lambda$-wavelength). In this case electrical field $\overline{\boldsymbol{E}}$ is markedly inhomogeneous and has two components $E_{\Theta}$ and $E_{r}$ (see Fig. 1). Components $E_{\Theta}$ and $E_{r}$ are not equal constant in the enclosed surface. The output RF voltage from the sensor, which is located on the "Enclosed surface (volume)" having its size, not be proportional to amplitude of the electrical field vector (or its components $E_{\Theta}$ and $E_{r}$ ). RF voltage will be proportional averaged field amplitude through sensor volume „V“. This article provides an answer to the question: What is dependence between the error of the spot metering and the size (volume) of the electrical field sensor?

\section{NEAR FIELD IN THE VICINITY OF THE SHORT DIPOLE}

Consider an electromagnetic field radiated by an electric dipole, which dimensions $2 h$ are much smaller than the wavelength $\lambda$ of the RF signal input. To simplify a mathematical description, the dipole is positioned symmetrically at the origin of the coordinate system and oriented along $z$ axis, as shown in Figure 1. The dipole is supplied by RF current $I$ with a frequency $\omega$. In this case we can easily, accurately and exactly define three components of the electromagnetic field and amplitude of the electromagnetic field (Vavra, 1989):

$$
\begin{aligned}
& E_{\theta}=-j \frac{2 h I k^{3} \sin \Theta}{4 \pi \omega \varepsilon}\left(\frac{1}{(k r)^{3}}+\frac{j}{(k r)^{2}}-\frac{1}{k r}\right) e^{-i k r} \\
& E_{r}=-j \frac{4 h I k^{3} \cos \Theta}{4 \pi \omega \varepsilon}\left(\frac{1}{(k r)^{3}}+\frac{j}{(k r)^{2}}\right) e^{-j k r} \\
& H_{\Phi}=\frac{2 h I k^{2} \sin \Theta}{4 \pi}\left(\frac{1}{(k r)^{2}}+\frac{j}{k r}\right) e^{-j k r}
\end{aligned}
$$

We can see that in the near area of the dipole (source dipole) two components of the electrical field (1), (2) exist. These components are orthogonal to each other; first one $E_{\theta}$ is oriented tangentially and the second one $E_{r}$ is oriented radially to the source dipole. If we want to measure the field in the near area of the dipole, we must measure each component separately (see Fig.2) and we get measurement result following:

$$
E=\sqrt{E_{\Theta}^{2}+E_{r}^{2}}
$$

Measuring of the each components of the field in the practical realization leads to a problem with measurement of radiation characteristic. The dimension of the sensors, which measure field, cannot be omitted and therefore we cannot talk about the spot metering. In this concept we can only use an approximation by the maximum reduction of the sensors size. 
Mostly electrically short dipoles or dipoles with a traveling wave are used to the electrical part of the electromagnetic field measure. "In such cases, it is necessary to get the maximal and minimal values of error of antenna factor at different angles of incidence, which are dependent on the antenna height over the ground plane" (Bittera \& Smieško, 2012). The length of an electrical short dipole is $L \ll \lambda$, and of the dipole with traveling wave is $L \sim \lambda$. In the both cases the output voltage is proportional to that part of the electrical field, which is tangential to the axis of the measuring dipoles, and length dipoles (Vavra, 1989). If the amplitude of the tangential component of the electric field is no constant on the length $L$, then output voltage of the sensor is described by the equation:

$$
U_{i}=\frac{1}{L} C \int_{-L / 2}^{L / 2} E(l) d l
$$

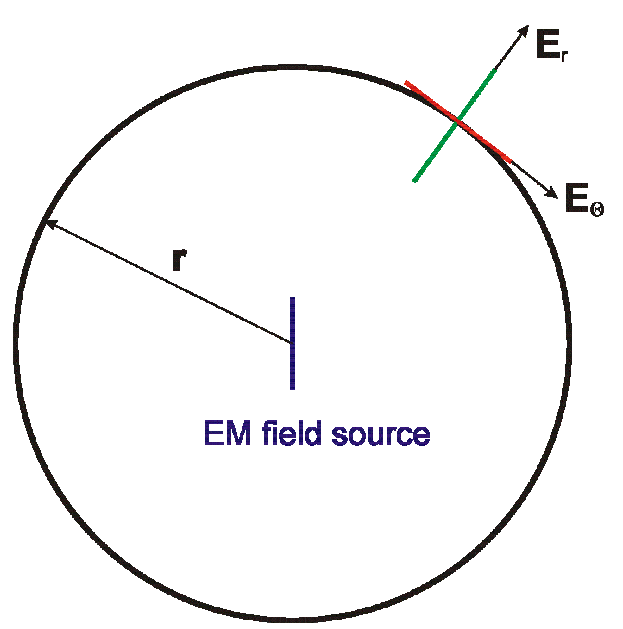

Figure 2 - Measurement of two components of the electric field

where $C$ is a constant given by properties of the sensor and $L$ is length of the sensor. Equation (5) describes the principle of the averaging field amplitude measurement. If the $L \rightarrow 0$, then output voltage of the sensor we, our equation is changed into (6) :

$$
U_{i}=C \cdot E(r, \theta)
$$

carries information about spot $M[r, \theta]$ metering amplitude of the electric field. In practice, such a case cannot occur, because infinitesimal length of the sensor has zero output voltage (Vavra, 1989). In this analysis, each measuring of the electrical component of the electromagnetic field is burden with methodological error. "Measurement is the process of extracting factual findings comparing entities with its purpose motivated sample in the surrounding area and time" (Zgodavova, 2010). To express the amplitude of this error, we must proceed separately with component $E_{\theta}$ and component $E_{r}$. Relative error measurement of component $E_{\theta}$ depends on the angle $\theta$ and a distance $r$ from source dipole (see Fig.3a): 


$$
\delta E_{\Theta}(r, \theta)=\frac{E_{\theta M E R}\left(r, \theta_{b}-\theta_{a}\right)-E_{\theta}(r, \theta)}{E_{\theta}(r, \theta)} \cdot 100 \%
$$

where $E_{\theta M E R}(r, \theta)$ is field amplitude which is obtained by dipole sensor, whose center is located at the place $[r, \theta]$ :

$$
E_{\text {बMER }}\left(r, \theta_{b}-\theta_{a}\right)=\frac{1}{\theta} \int_{\theta_{a}}^{\theta_{b}} E_{\theta}(r, \theta) d \theta=\frac{r}{L} \int_{\theta-\frac{L}{2 r}}^{\theta+\frac{L}{2 r}} E_{\theta}(r, \theta) d \theta
$$

and $E_{\theta}(r, \theta)$ represents ideal amplitude of the electric field component, calculated according to (1) in point of $M[r, \theta]$. Equation (7) was obtained from the definition of the relative error of measurement. Equation (8) is modified (5) without constant $C$, which represents properties of the dipole sensor. Because the electric field varies along $\theta$ axis, interval of integration (8) will be in radian.

Relative errors of component $E_{r}$ is calculated similarly. In this case, the electric field component is depending on the position $r$ of dipole sensor and on its length L. (see Fig.3b), then we get:

$$
\delta E_{r}(r, \Theta)=\frac{E_{r M E R}\left(r_{b}-r_{a}, \Theta\right)-E_{r}(r, \Theta)}{E_{r}(r, \Theta)} \cdot 100 \%
$$

In equation (9) $E_{r M E R}(r, \theta)$ is field amplitude which is obtained by the dipole sensor, whose center is located at the place $[r, \theta]$ :

$$
E_{r M E R}\left(r_{b}-r_{a}, \Theta\right)=\frac{1}{r} \int_{r_{a}}^{r_{b}} E_{r}(r, \theta) d r=\frac{1}{L} \int_{r-\frac{L}{2}}^{r+\frac{L}{2}} E_{r}(r, \theta) d r
$$

in (10) and (8) are different in view of the location of the measuring dipole sensors (see Fig.3).

To determine total measurement error from components $E_{\theta}$ and $E_{r}$ we cannot use the error propagation law in indirect measurement of value - it is a systematic error. "Result of measurement is measured value. This value is loaded with error, which characterized precision, accuracy and quality of measurement" (Bittera, et al., 2012). We must express the total electric field using the equation: (1), (2), (4):

$$
E(r, \Theta)=\sqrt{E_{\theta}^{2}(r, \Theta)+E_{r}^{2}(r, \Theta)}
$$




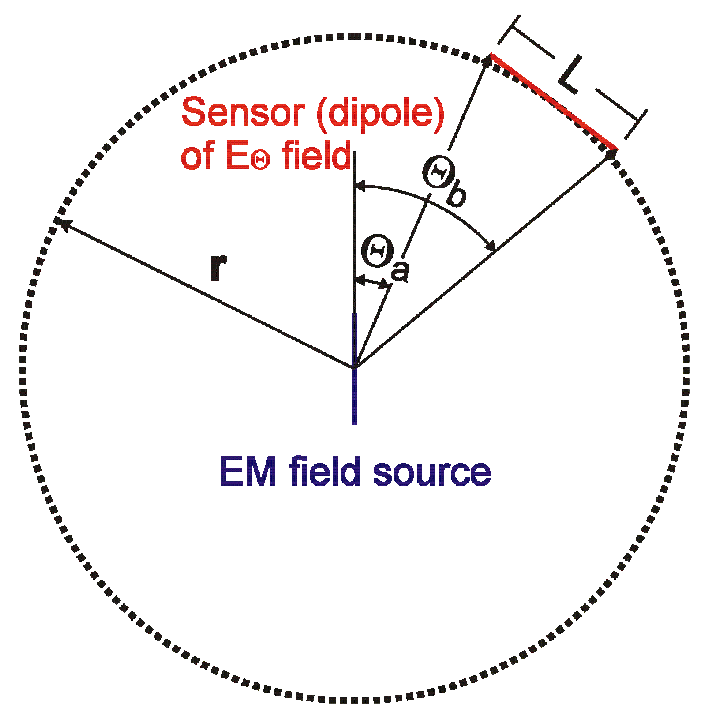

a)

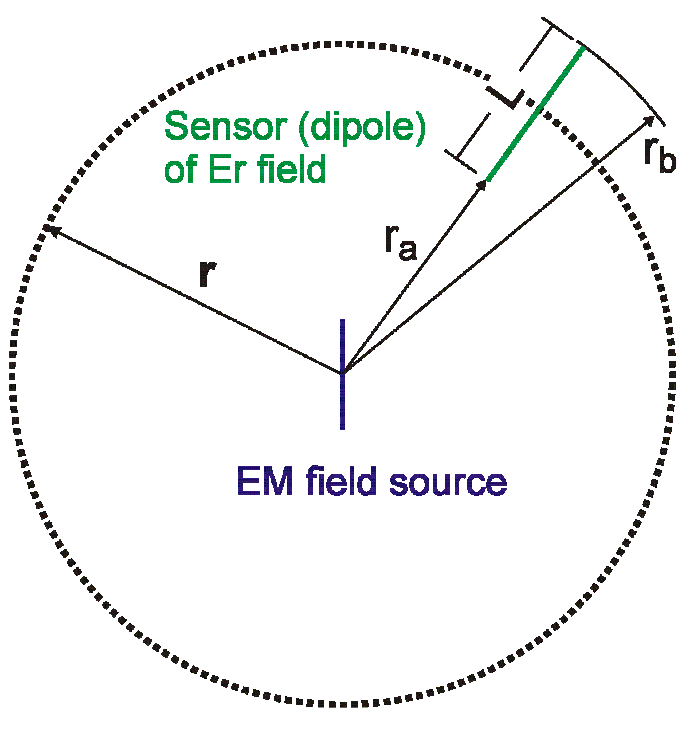

b)

Figure 3 - Measurement of tangential and radial components of the electric field

and total electric field (11) using measure (average calculation) (5) applied to the (1), (2):

$$
E_{\text {MER }}(r, \Theta)=\sqrt{\left(\frac{r}{L} \int_{\theta-\frac{L}{2 r}}^{\theta+\frac{L}{2 r}} E_{\theta}(r, \theta) d \theta\right)^{2}+\left(\frac{r}{L} \int_{\theta-\frac{L}{2 r}}^{\theta+\frac{L}{2 r}} E_{\theta}(r, \theta) d \theta\right)^{2}}
$$

Then we get expression of the total relative error:

$$
\delta E(r, \Theta)=\frac{E_{M E R}(r, \Theta)-E(r, \Theta)}{E(r, \Theta)} \cdot 100 \%
$$

\section{RESULTS OF ANALYTICAL SOLUTION}

The near field calculation was performed for the electrically short dipole with a length of $0.1 \mathrm{~m}$, supplied by a signal with frequency of $10 \mathrm{MHz}$ and current amplitude $1 \mathrm{~A}$. In the Fig. 4 we can see waveforms of intensity $E_{\theta}(r, \theta)$ and $E_{r}(r, \theta)$, obtained by using equation (1) and (2). Radial and tangential field have $|\operatorname{Sin}(\theta)|$ respectively $|\operatorname{Cos}(\theta)|$ function shape in the space, while the fields are shifted each other of $\pi / 2$ in the direction $\theta$ and $r=0.2995 \mathrm{~m}$. 

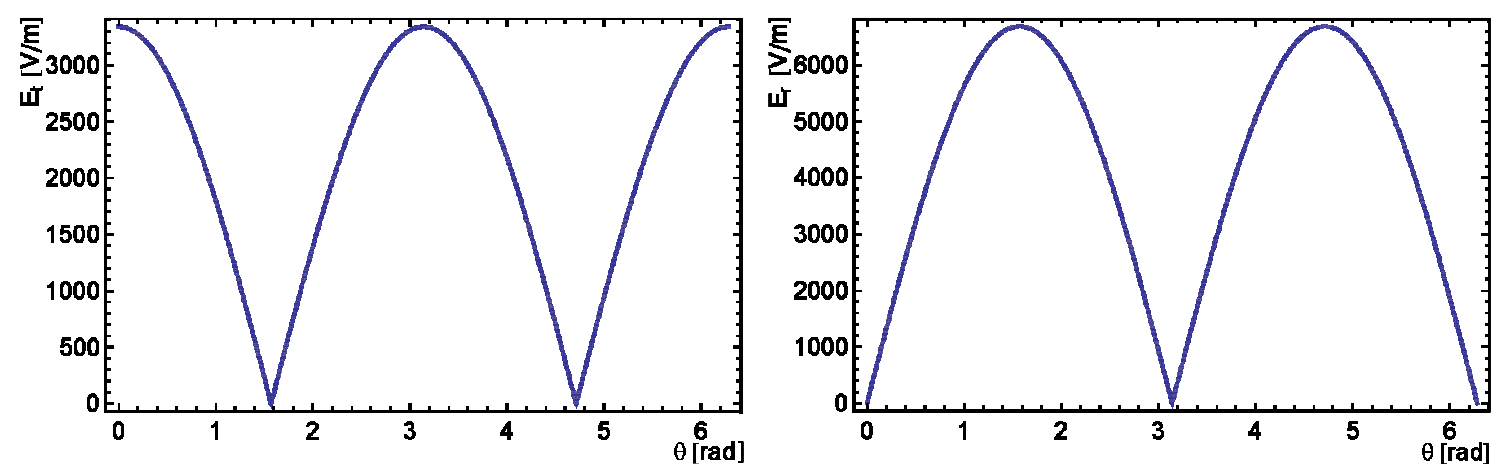

Figure 4 - Near field shape of the electrically short dipole

In the near field $k r<<1$ the radial component of the electric field is dominated (see Fig. 4). If the individual components of the electric near field is measured by sensors (dipole antennas), which have real dimensions, we will get the results burdened by error. Shape of the measured radiation characteristics will be different in comparison with the reality. The measurement average effect was simulated using equations (1), (2) and (5). The result for the distance from the source dipole $r=0.2995 \mathrm{~m}$ and sensor length $L=r / 2$ (on frequency $10 \mathrm{MHz}$ the $L / \lambda=0.79610^{-3}$ ) are in the Fig.5.
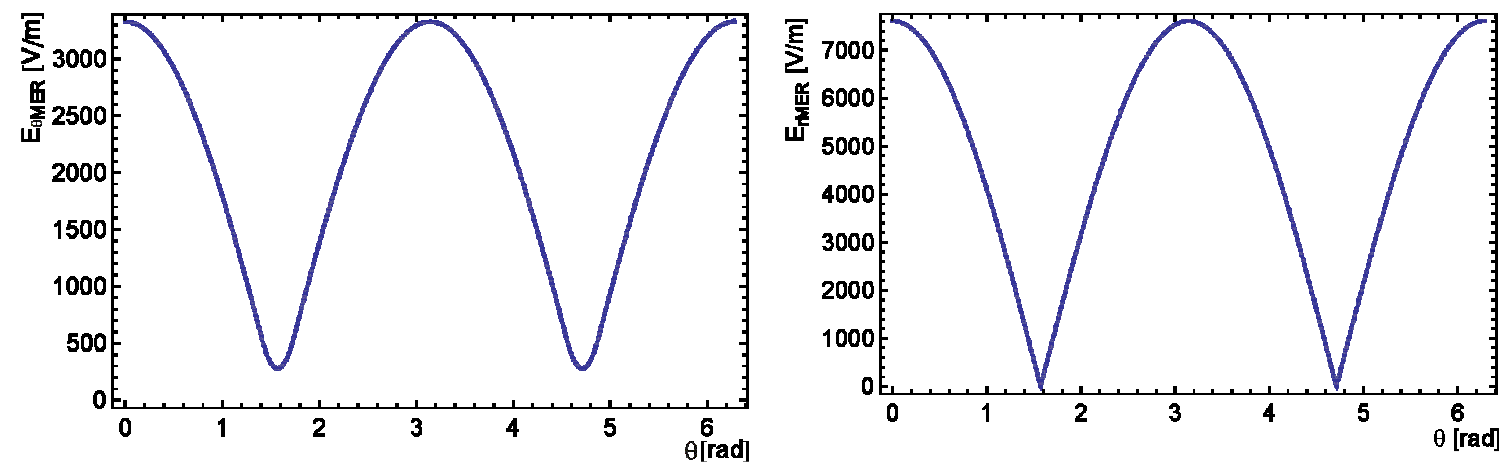

Figure 5 - Near field shape of the electrically short dipole using simulated measure by the sensor with dimension $L=r / 2=0.14975 m$ (average calculation)

Since we know shapes of two components of the electric near field in the close of the electrically short dipole, we can calculate the total electric field by equation (4). This calculation we can carried out with the ideal values of the electric field, as well as the average (simulated measure) values of the electric field. Distributions of the electric near field of the electrically short dipole are showed, in Fig.6. These graphs are obtained by the equations (1), (2), (4), (5). 

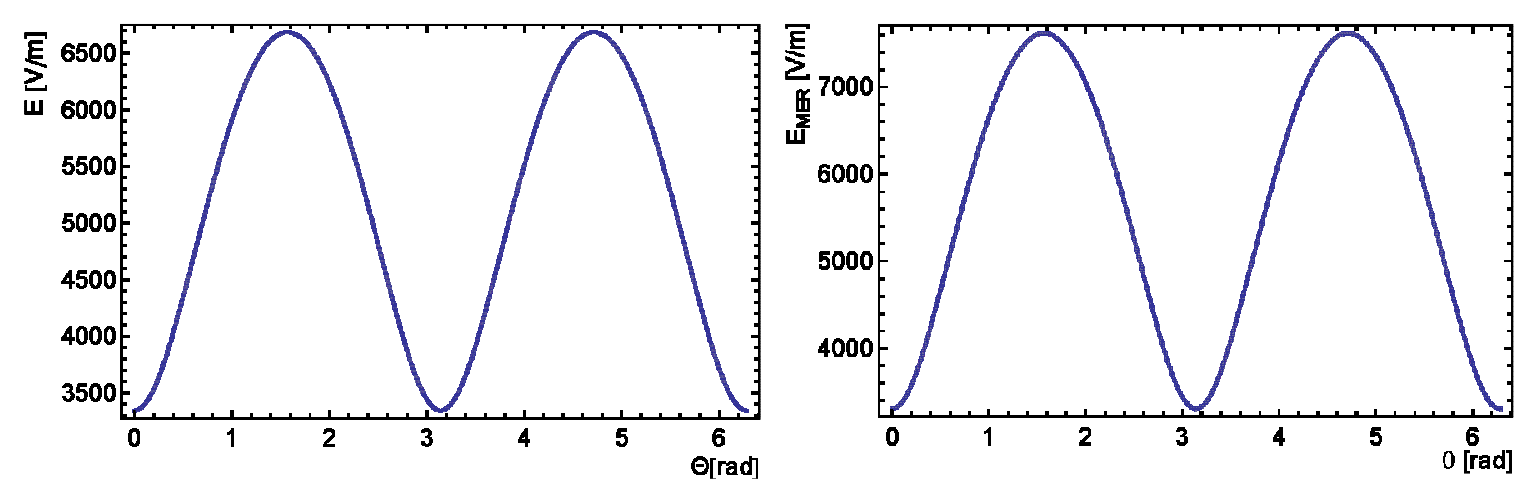

Figure $6-a)$ Near field shape of the ideal electric electrically short dipole b) Near field shape of the electric electrically short dipole obtained by simulated measure

Comparing the ideal and simulated fields and using (13) we get graphs of the systematic errors which are expressed in percentage. Comparison for different length of the sensor is in Fig.7.

Based on the Fig.7 we can see that measurement error of the near field is decreased by the reducing the size of the sensor (measuring dipole). The maximum value $+15 \%$ is reached when dimension of sensor are $L=r / 2$ (Fig.7 left, line). This value is achieved if the sensor length is half of the distance from measured source, it is 0.0008 of wavelength. The case of the sensor whose dimension is $L=r / 3$ (Fig.7 left, dashed line) error of measurement reached the maximum value $+6 \%$. Then sensor size is one third of the distance from measured source, it is 0.0005 of wavelength. The analyzed data (Fig. 7 right) indicate that the size of the sensor must be significantly less than the wavelength of the measured electric field.
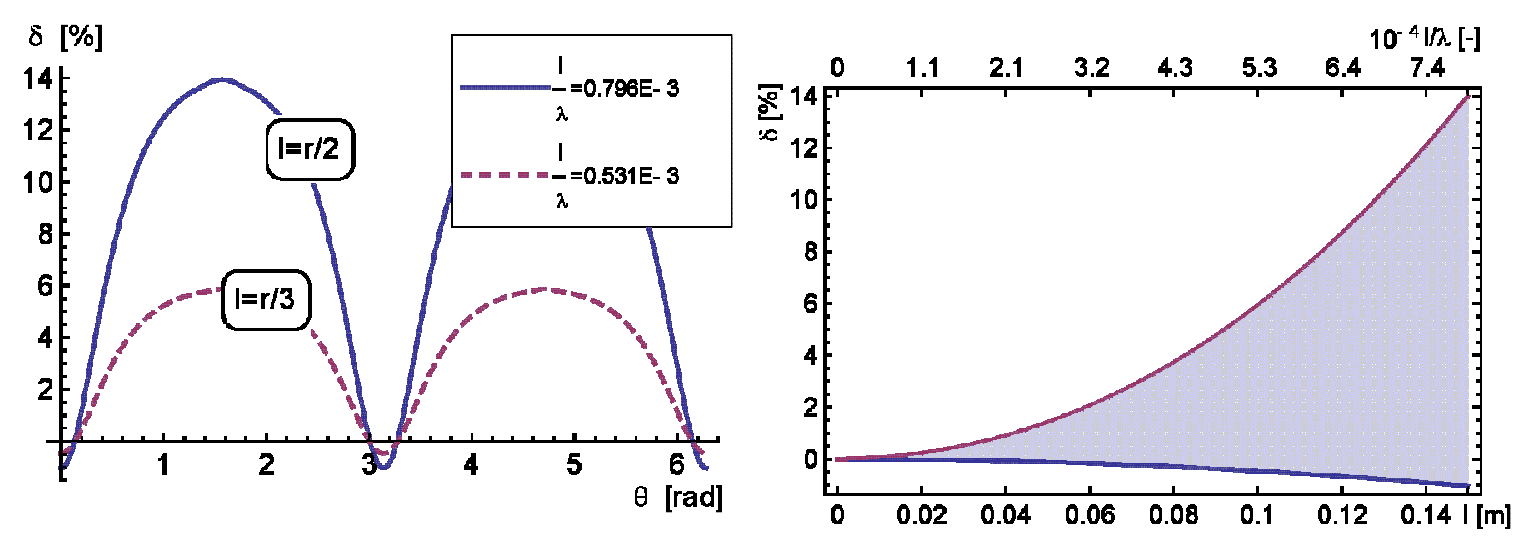

Figure 7 - Relative systematic errors for measured near field of electrically short dipole - analytical calculation 


\section{NUMERICAL VERIFICATION OF SOLUTIONS}

To prove derived analytical solution of the systematic error it is necessary to perform measurement or numerical simulation of the problem. Because measurement can bring into the result additional errors, we chose numerical simulation. Was be used method of moments (MoM) for numerical simulation, because dipole can be represented by wire structures.

There are a lot of programs that enable solving such a problem. FEKO solver have implemented the moment method and allows us solve wide range of the electromagnetic problem. The source dipole was modeled as an ideal wire with a diameter of $1 \mathrm{~mm}$ and a length of $10 \mathrm{~cm}$. It was supplied by a signal with a frequency of $10 \mathrm{MHz}(l<\lambda$ - equation (1-3) are satisfied) and voltage $1 \mathrm{~V}$. In the distance of $r=r_{0}$ for each point $\left[r_{0}, \theta\right]$ components of near field $E_{\theta N}(r, \theta)$ and $E_{r N}(r, \theta)$ were numerically calculated and expressed their combination according to (4).

The real electric field measurement process was modeled by dipole with length $L$ was placed into the point $\left[r_{0}, \theta\right]$, see Fig.3. The inducted voltage at dipole terminals was numerically calculated. From this voltage was obtained the field $E_{\theta N M E R}(r, \theta)$ or $E_{r N M E R}(r, \theta)$, respectively.

To verify the analytical calculation of the near-field measurement errors, we performed a numerical simulation in FEKO. Geometry of the sensor and source dipole was the same as in the analytical model (source dipole length $0.1 \mathrm{~m}$, distance from source dipole $r=0.2995 \mathrm{~m}$ and sensing dipole length $L=r / 3$, the frequency of the power source was $10 \mathrm{MHz}$ ). The result of numerical analysis were terminal voltages

$$
U_{\theta N}(r, \theta)=f\left(E_{\theta N M E R}(r, \theta)\right)
$$

and

$$
U_{r N}(r, \theta)=f\left(E_{r N M E R}(r, \theta)\right)
$$

Comparison of the relative error obtained by analytical and numerical methods are illustrated in the Fig.8. We can see that the curve shape is almost identical, even though they are mutually shifted in the axis , $y$ ". The resulting shift can be caused by evaluation of numerical simulation - imperfect calculation inverse function of (14), (15). Also we have done simplification in the analytical expression of the systematic error. We assumed the tangential configuration $E_{\theta}$ of the sensor field - dipole antenna related to measuring electromagnetic field component $\theta$. In general, numerical simulation confirmed the theoretical assumptions of the equation (5). 


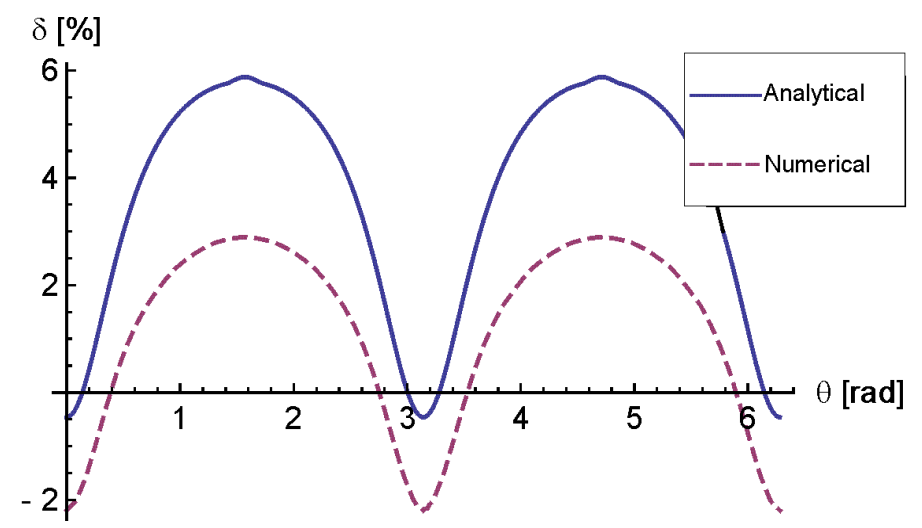

Figure 8 - Comparison of numerical and analytical results

\section{CONCLUSION}

In process of measurement of the near field a several errors brings in to the measurement results. In particular it is the frequency error of the sensor, which is reflected on the border of its frequency band and errors of the measured values averaging. In this paper we answer to the question, how the averaging errors reflected on the measurement result of the electric near field. The generally accepted theories recommend length of the sensor (measuring dipole) $L=\lambda / 10$. At this length we ensure the minimum errors resulting from averaging the measured values, only for far field. In the near field, these theories do not apply, because the shape of the field varies depending on the distance from the source and position of the observation point. The near field is strongly inhomogeneous field. Of course, the shape of the near field is affected by the wavelength, but this effect is much less important than influence of dimension $r$ and $\theta$. The results of the analysis are the length of sensor $L$, which is one thousandth of wavelength depending on the required measurement accuracy (1\% error) of the near field $L=6.4 \lambda / 1000$. Scientific contribution of the article is quantifying errors of measurement of the electric field. It is presented relation between the dimensions of the measuring sensor and averaging error of the electric field. The practical contribution is alignment of the metrological laboratories so that the during the measurement of the electromagnetic field is the measurement uncertainty caused by methodological errors reduced.

\section{ACKNOWLEDGEMENT}

This work was supported by the Slovak Research and Development Agency under the contract No. APVV-0333-11 Electromagnetic compatibility of technology in rubber industry and by the project VEGA 1/0963/12 Validation methods of selected test of EMC. 


\section{REFERENCES}

Hart'anský, R., Bittera, M., 2001. Assurance of non-directive pattern of electromagnetic field sensor. Mechatronika $2001-4^{\text {th }}$ International Symposium Mechatronics. Jun 2001. Trenčianské Teplice: TnUAD.

Vavra, Š., 1989. Antény a šírenie elektromagnetických vín. Bratislava: Alfa.

Bittera, M., Kamenský, M., Králiková, E., 2012. Elektrické meranie: Návody na laboratórne cvičenia. Bratislava: STU v Bratislave FEI.

Zgodavova, K., 2010. Complexity of entities and its metrological implications", 0365-0367, Annals of DAAAM for 2010 \& Proceedings of the $21^{\text {st }}$ International DAAAM Symposium, ISBN 978-3-901509-73-5, ISSN 1726-9679, pp 0183, Editor B. Katalinic, Published by DAAAM International, Vienna, Austria 2010;

Petrik, J., Palfy, P., 2009. The quality of hardness tester calibration. Quality Innovation Prosperity XIII/1- 2009. 13(1).

Bittera, M., Smieško, V., 2012. Directivity Error of Broadband Antennas at EMI Measurement. Przeglad elektrotechniczny. 88(2).

\section{ABOUT THE AUTHOR}

Jozef Slížik received master (Ing.) degree in measurement and information technology from Faculty of Electrical Engineering and Information Technology of the Slovak Technical University (FEI STU) in 2012. He is currently a PhD. student at the Faculty of Electrical Engineering and Information Technology of the STU Bratislava.; e-mail: xslizik@ stuba.sk.

René Hart'anský (doc., Ing., PhD.) received the master (Ing.) degree in 1992 and then $\mathrm{PhD}$. degree in measurement technique from the Faculty of Electrical Engineering of the Slovak Technical University (FEE STU) in 1999. He is currently the associate professor (docent) at the Institute of Electrical Engineering of the STU Bratislava. His research interests are the computer modeling EMC phenomena, antennas and propagation, electromagnetic field metrology; e-mail: rene.hartansky@stuba.sk. 